\title{
Permutations avoiding two patterns of length three
}

\author{
Vincent R. Vatter* \\ Department of Mathematics \\ Rutgers University, Piscataway, NJ 08854 \\ vatter@math.rutgers.edu
}

Submitted: Nov 25, 2002; Accepted: Jan 9, 2003; Published: Jan 22, 2003

MR Subject Classifications: 05A15, 68R15

Keywords: Restricted permutation, forbidden subsequence, generating tree

\begin{abstract}
We study permutations that avoid two distinct patterns of length three and any additional set of patterns. We begin by showing how to enumerate these permutations using generating trees, generalizing the work of Mansour [13]. We then find sufficient conditions for when the number of such permutations is given by a polynomial and answer a question of Egge [6]. Afterwards, we show how to use these computations to count permutations that avoid two distinct patterns of length three and contain other patterns a prescribed number of times.
\end{abstract}

\section{Introduction}

Let $q=q_{1} q_{2} \ldots q_{k}$ be a permutation in the symmetric group $S_{k}$. We call $k$ the length of $q$ and write $|q|=k$. The reduction of a word $w$ of distinct integers of length $k, \operatorname{red}(w)$, is the $k$-permutation obtained by replacing the smallest number element of $w$ by 1 , the second smallest element by 2 , and so on. We say that the permutation $p=p_{1} p_{2} \ldots p_{n} \in S_{n}$ contains a q pattern if there is a subsequence $p_{i_{1}} p_{i_{2}} \ldots p_{i_{k}}$ of $p$ that reduces to $q$, that is, $\operatorname{red}\left(p_{i_{1}} p_{i_{2}} \ldots p_{i_{k}}\right)=q$. Otherwise we say that $p$ is $q$-avoiding. For example, 3142 contains a 132 pattern because $\operatorname{red}(142)=132$, whereas 3124 is 132 -avoiding.

Let the set $S_{n}(q)$ consist of all $n$-permutations that avoid $q$. If $Q$ is a set of permutations, we define

$$
S_{n}(Q)=\bigcap_{q \in Q} S_{n}(q)
$$

*This work has been partially supported by an NSF VIGRE grant to the Rutgers University Department of Mathematics. 
so $S_{n}(Q)$ consists of all $n$-permutations that avoid every member of $Q$. We also define

$$
S(Q)=\bigcup_{n \geq 1} S_{n}(Q)
$$

and set

$$
s_{n}(Q)=\left|S_{n}(Q)\right| .
$$

Note that if $q_{1}, q_{2} \in Q$ and $q_{2}$ contains $q_{1}$, then the $q_{2}$ restriction is superfluous, since every $q_{1}$-avoiding permutation is also $q_{2}$-avoiding. Hence we may assume that $Q$ is an antichain with respect to the pattern containment ordering.

The problem of finding the cardinality of $S_{n}(q)$ for various patterns $q$ has received much attention. The first two calculations were $s_{n}(123)$ and $s_{n}(132)$, by MacMahon [12] and Knuth [10] respectively. Both cardinalities turn out to be the $n$th Catalan number. Later, Simion and Schmidt [18] found $s_{n}(Q)$ for all $Q \subseteq S_{3}$. This was followed by several articles that found $s_{n}\left(\left\{q_{1}, q_{2}\right\}\right)$ for various pairs of permutations: Billey, Jockusch, and Stanley [4], Guibert [9], and West [19] solved the problem for $q_{1} \in S_{3}, q_{2} \in S_{4}$, and Kremer and Shiu [11] did several cases with $q_{1}, q_{2} \in S_{4}$.

Two recent articles articles have dealt with counting permutations that avoid at least two patterns of length three subject to other constraints. Mansour [13] found the generating functions for $s_{n}(Q \cup\{q\})$ explicitly (in the form of a determinant) for all patterns $q$ and sets $Q \subset S_{3}$ with $|Q| \geq 2$. Later, Mansour [14] computed generating functions for the number of permutations that avoid at least two patterns of length three and contain another pattern (of any length) exactly once. We generalize and combine these results in this paper.

We will start by showing how to routinely find $s_{n}(Q)$ for all sets of permutations $Q$ with $\left|Q \cap S_{3}\right| \geq 2$. Using ideas from Atkinson [1], we go on to show that this gives us an algorithm to find the number of $n$-permutations that avoid two patterns of length three and contain a finite set of other patterns a prescribed number of times. Along the way, we answer a question of Egge [6] and see when the level sums of a generating tree agree with a polynomial.

We begin with definitions. If $q$ is a permutation and $q^{-1}$ is its group-theoretic inverse, then by elementary arguments (see, for example, Simion and Schmidt [18]), $s_{n}(q)=$ $s_{n}\left(q^{-1}\right)$ for all $n$. The same holds between $q$ and its reverse, $q^{\text {rev }}$, where $q^{\text {rev }}(i)=q(|q|+$ $1-i$ ). These two operations generate the dihedral group of order 8. If $Q_{2}$ is a set of permutations that can be obtained from $Q_{1}$ by an element of this group, then $s_{n}\left(Q_{1}\right)=$ $s_{n}\left(Q_{2}\right)$ and we say that $Q_{1}$ and $Q_{2}$ are in the same symmetry class.

If $Q_{1}$ and $Q_{2}$ are sets of patterns with $s_{n}\left(Q_{1}\right)=s_{n}\left(Q_{2}\right)$ for all $n$ then we say that $Q_{1}$ and $Q_{2}$ are Wilf-equivalent, or that they belong to the same Wilf class. As is the case with 123 and 132, it can happen that two patterns are Wilf-equivalent even though they are not in the same symmetry class. One of the advantages of our approach is that is makes Wilf-equivalence particularly easy to notice (see Corollaries 3.4, 3.7, and 3.9). 
There are only six symmetry classes of two element subsets of $S_{3}$, listed below.

\begin{tabular}{c|c} 
symmetry class & members \\
\hline \hline$A$ & $\{132,231\},\{213,312\},\{132,312\},\{213,231\}$ \\
\hline$B$ & $\{132,213\},\{231,312\}$ \\
\hline$C$ & $\{123,132\},\{123,213\},\{231,321\},\{312,321\}$ \\
\hline$D$ & $\{132,321\},\{123,231\},\{123,312\},\{213,321\}$ \\
\hline$E$ & $\{123,321\}$
\end{tabular}

Simion and Schmidt [18] found that these sets form only three Wilf classes. In particular, they showed that $s_{n}(Q)=2^{n-1}$ if $Q$ belongs to any of the symmetry classes $A$, $B$, or $C, s_{n}(Q)=1+\left(\begin{array}{l}n \\ 2\end{array}\right)$ if $Q$ belongs to class $D$, and for $n \geq 5, s_{n}(Q)=0$ if $Q$ is the set in class $E$. For the remainder of this article we ignore the degenerate $\{123,321\}$ case. We rederive the other results in the next section because we will need to know more than just the cardinality of $S_{n}(Q)$.

\section{Generating trees}

Our results will make use of what are known as generating trees. The introduction of generating trees is due to Chung et al. [5], who used them to count Baxter permutations and recommended their use in other problems involving permutations. Recently many authors have followed this advice. The reader is referred to West's papers [19] and [20] for numerous examples and references. More generally, several authors have begun to study the algebraic properties of generating trees, see Banderier et al. [3], Ferrari et al. [7], and the references therein.

Precisely, a generating tree is a rooted, labeled tree such that the labels of the children of a node are determined by the label of that node. Therefore we specify a generating tree by providing the label of the root and a set of succession rules. For example, the complete binary tree is given by

$$
\begin{aligned}
& \text { Root: }(2) \\
& \text { Rule: }(2) \leadsto(2)(2)
\end{aligned}
$$

If $T$ is a tree, we will let $T_{\leq x}$ denote the subtree of $T$ containing $x$ and all of its descendants. Also, because it agrees with our applications to permutations, we will say that the root of $T$ is on level 1 , and for any level $n$, we will refer to the number of nodes on level $n$ as the $n$th level sum of $T$.

To use generating trees to calculate $s_{n}(Q)$ for a set of patterns $Q$, we first build the tree $T(Q)$ (which we will call a pattern-avoidance tree) with nodes $S(Q)$ where $p \in S_{n}(Q)$ 
is a child of $p^{\prime} \in S_{n-1}(Q)$ if $p$ is formed by inserting $n$ somewhere in $p^{\prime}$. Then, we find a generating tree that is isomorphic to $T(Q)$. Four easy examples are contained in the next two propositions. Certainly these results are not original, but it seems that the derivation of $T(\{132,231\})$ is the only one that has appeared in the literature (in West [19]).

Proposition 2.1 The pattern avoidance trees $T(\{312,321\}), T(\{132,213\})$, and $T(\{132,231\})$ are all isomorphic to the complete binary tree, so if $Q$ belongs to class $A$, $B$, or $C$, then $s_{n}(Q)=2^{n-1}$.

Proof: We will need a separate ad hoc argument for each tree. First, if $n \geq 3$ and $p \in S_{n-1}(\{312,321\})$, then clearly we cannot insert $n$ anywhere before the second-to-last element of $p$, since the last two elements of $p$ either form a 12 pattern or a 21 pattern. Furthermore, the insertion of $n$ into either the next-to-last or last position in $p$ must produce a permutation in $S(\{312,321\})$ because there will not be enough elements after $n$ to create a new 312 or 321 pattern. Therefore each node of $T(\{312,321\})$ has precisely two children, as desired.

Now assume $p \in S_{n-1}(\{132,213\})$. We cannot insert $n$ anywhere to the left of $n-1$, unless we insert $n$ at the very beginning, because otherwise we create a 132 pattern. Also, to avoid creating a 213 pattern, we cannot insert $n$ anywhere after $n-1$ unless we insert $n$ immediately after $n-1$. It is easily checked that both of these insertions are fine, so again every node of $T(\{132,213\})$ has precisely two children.

For the last case, let $p \in S_{n-1}(\{132,231\})$. We can insert $n$ at the beginning or end of $p$, and nowhere in between, completing the proof.

Proposition 2.2 The pattern avoidance tree $T(\{132,321\})$ is isomorphic to the generating tree given by

$$
\begin{array}{ll}
\text { Root: } & (\overline{2}) \\
\text { Rules: } & (\overline{2}) \leadsto(2)(\overline{2}) \\
& (2) \leadsto(2)(1) \\
& (1) \leadsto(1)
\end{array}
$$

so if $Q$ is a member of class $D, s_{n}(Q)=\left(\begin{array}{l}n \\ 2\end{array}\right)+1$.

Proof: Let $p \in S_{n-1}(132,321)$. If $p=12 \ldots(n-1)$, then we may insert $n$ at the beginning or end of $p$, but nowhere in between; these permutations correspond to nodes labeled $(\overline{2})$. If $p \neq 12 \ldots(n-1)$, we cannot insert $n$ at the very beginning of $p$ because that would create a 321 pattern, and we cannot insert $n$ anywhere else before $n-1$ because that would create a 132 pattern. We can insert $n$ right after $n-1$ or at the end of $p$ (in some cases these two positions are the same, and this is when $p$ corresponds to a node labeled (1)). Furthermore, we cannot insert $n$ elsewhere after $n-1$, because that would create a 132 pattern (since $n-1$ was not involved in a 321 pattern). $\diamond$ 


\section{Tree pruning and Wilf-equivalence}

When $Q$ contains at least two patterns of length three, $T(Q)$ is a subtree of $T\left(Q \cap S_{3}\right)$. Of course, not every subtree is possible. For example, $T(Q)$ cannot be isomorphic to $T(\{132,312\})$ with just the branch rooted at 12 cut off, because that would imply that $12 \in Q$, and thus other branches would need to be pruned as well. Our goal is to discover a set of "pruning rules" that will tell us in what ways these trees can be pruned. These pruning rules will reduce the problem of enumerating permutations that avoid a set of patterns to the much easier problem of enumerating words that avoid (in a few different senses) a set of subwords. Although $T(\{132,231\}) \cong T(\{132,213\}) \cong T(\{312,321\})$, we will see that each tree prunes differently. We start with the easiest tree to prune, $T(\{132,231\})$.

Given an alphabet $A$, let $A^{n}$ stand for the set of all words of length $n$ with letters from $A$ and let $A^{*}=\cup_{n} A_{n}$ denote the set of all finite words over $A$. If $w \in A^{n}$, we let $|w|=n$. We denote the empty word by $\epsilon$. If $u$ and $w=\ell_{1} \ell_{2} \ldots \ell_{n}$ are both words, where $\ell_{i} \in A$ for all $1 \leq i \leq n$, we write $u \preceq w$ if and only if $w$ contains $u$ as a (not necessarily contiguous) subword, i.e., if and only if there is a set of indices $i_{1}<i_{2}<\ldots<i_{k}$ such that $\ell_{i_{1}} \ell_{i_{2}} \ldots \ell_{i_{k}}=u$.

We associate with each permutation $p \in S_{n}(\{132,231\})$ a word $w_{A}(p) \in\{L, R\}^{n-1}$ in the following recursive manner. First, we set $w_{A}(1)=\epsilon$. For $n>1$, assume that $p$ is formed by inserting $n$ into $p^{\prime}$. Let $w_{A}(p)=w_{A}\left(p^{\prime}\right) L$ if $p(1)=n$ and $w_{A}(p)=w_{A}\left(p^{\prime}\right) R$ if $p(n)=n$ (by Proposition 2.1 these are the only two possibilities).

Theorem 3.1 Let $p, q \in S(\{132,231\})$. Then $p$ contains a $q$ pattern if and only if $w_{A}(q) \preceq w_{A}(p)$.

Proof: Let $n=|p|$ and $k=|q|$. We induct on $n$. If $n=1$ then $p=1$ and the theorem is easily verified. Similarly, we may assume that $k>1$, so there are (possibly empty) words $w$ and $w^{\prime}$ and letters $\ell, \ell^{\prime} \in\{L, R\}$ so that $w_{A}(q)=w \ell$ and $w_{A}(p)=w^{\prime} \ell^{\prime}$. Hence $q$ is formed by inserting $k$ into $w_{A}^{-1}(w)$ and $p$ is formed by inserting $n$ into $w_{A}^{-1}\left(w^{\prime}\right)$.

First, assume that $p$ contains a $q$ pattern. Then $w_{A}^{-1}\left(w^{\prime}\right)$ contains a $w_{A}^{-1}(w)$ pattern, so by induction, $w \preceq w^{\prime}$. If $w_{A}(q) \preceq w^{\prime} \preceq w_{A}(p)$ then we are done, so we may assume that $w_{A}(q) \npreceq w^{\prime}$. Then by induction every $q$ pattern in $p$ uses the element $n$, so since this element must play the role of $k$ in any $q$ pattern it participates in, $\ell=\ell^{\prime}$ as desired.

Now assume that $w_{A}(q) \preceq w_{A}(p)$, so $w \preceq w^{\prime}$. If $w_{A}(q) \preceq w^{\prime}$, then we are done by induction. Hence we may assume that $\ell=\ell^{\prime}$. By induction $w_{A}^{-1}\left(w^{\prime}\right)$ contains a $w_{A}^{-1}(w)$ pattern, and since $\ell=\ell^{\prime}$, either $q(1)=k$ and $p(1)=n$ or $q(k)=k$ and $p(n)=n$. In both cases we find a $q$ pattern in $p$, completing the proof. $\diamond$

The previous theorem allows us to easily construct generating trees isomorphic to $T(Q)$ for all $Q$ containing both 132 and 231. If $u, w \in\{L, R\}^{*}$ and $u=\ell_{1} \ell_{2} \ldots \ell_{k}$ where each $\ell_{i}$ is a letter, let

$$
m_{u}(w)=\max \left\{i: \ell_{1} \ell_{2} \ldots \ell_{i} \preceq w\right\}
$$


so $m_{u}(w)$ tells us how much of $u$ we have in $w$. Now set

$$
Q^{\prime}=Q \backslash\{132,231\}=\left\{q_{1}, q_{2}, \ldots, q_{r}\right\} .
$$

For convenience, let $w_{i}=w_{A}\left(q_{i}\right)=\ell_{i, 1} \ell_{i, 2} \ldots \ell_{i,\left|q_{i}\right|-1}$ where $\ell_{i, j} \in\{L, R\}$. By Theorem 3.1, we can associate with each $p \in S(Q)$ a vector

$$
\begin{aligned}
\vec{v}_{Q^{\prime}}(p) & =\left(m_{w_{1}}\left(w_{A}(p)\right)+1, m_{w_{2}}\left(w_{A}(p)\right)+1, \ldots, m_{w_{r}}\left(w_{A}(p)\right)+1\right) \\
& \in\left[\left|q_{1}\right|-1\right] \times\left[\left|q_{2}\right|-1\right] \times \ldots \times\left[\left|q_{m}\right|-1\right],
\end{aligned}
$$

because if $m_{w_{i}}\left(w_{A}(p)\right)=\left|q_{i}\right|-1=\left|w_{i}\right|$, then $w_{i} \preceq w_{A}(p)$ and thus $p \notin S(Q)$. If $\vec{a}$ is any such vector and $\ell \in\{L, R\}$, let $d_{\ell}(\vec{a})=\left(b_{1}, b_{2}, \ldots, b_{r}\right)$ where

$$
b_{i}:= \begin{cases}a_{i}+1 & \text { if } \ell_{i, a_{i}+1}=\ell, \\ a_{i} & \text { otherwise, }\end{cases}
$$

Then by Theorem 3.1, $T(Q)$ is isomorphic to the generating tree with labels $\left[\left|q_{1}\right|-1\right] \times$ $\left[\left|q_{2}\right|-1\right] \times \ldots \times\left[\left|q_{m}\right|-1\right]$ and root $\overrightarrow{1}=(1,1, \ldots, 1)$ in which for each $\ell \in\{L, R\}$, any node labeled $\vec{a}$ produces a child labeled $d_{\ell}(\vec{a})$ if and only if $d_{\ell}(\vec{a}) \in\left[\left|q_{1}\right|-1\right] \times\left[\left|q_{2}\right|-1\right] \times$ $\ldots \times\left[\left|q_{m}\right|-1\right]$.

Note that if $Q$ is a finite set of patterns, then the generating tree given above has only finitely many labels, and thus it is well-known that the generating function for $s_{n}(Q)$ is rational (and easily computed). In fact, since we have assumed that $Q$ is an antichain the following result of Atkinson et al. implies that $Q$ is finite. Recall that a partially ordered set is called partially well ordered if it contains neither an infinite strictly decreasing sequence nor an infinite antichain.

Theorem 3.2 [2] For all sets of patterns $Q$ with $\left|Q \cap S_{3}\right| \geq 2$, S(Q) is partially well ordered.

Furthermore, in Section 5, we will show that if $Q$ contains 132, 231, and at least one pattern from $S(\{132,231\})$, then $s_{n}(Q)$ is essentially a polynomial (we postpone the definition of "essentially" until Corollary 5.3).

Now we move on to the case of avoiding 132 and 213. As in the last case, for each $p \in S_{n}(\{132,213\})$ we recursively define a word $w_{B}(p)$ of length $n-1$. First set $w_{B}(1)=\epsilon$. For $n>1$, assume that $p$ is formed by inserting $n$ into $p^{\prime}$. By Proposition 2.1, we know that there are only two ways in which this insertion can be performed. If $p(1)=n$, set $w_{B}(p)=w_{B}\left(p^{\prime}\right) L$. Otherwise, $n$ was inserted right after $n-1$, and we set $w_{B}(p)=$ $w_{B}\left(p^{\prime}\right) R$.

If $u, w \in\{L, R\}^{*}$, we say that $w$ contains $u$ as a factor if $u$ occurs as a contiguous subword in $w$, that is, if there are (possibly empty) words $w_{1}, w_{2} \in\{L, R\}^{*}$ such that $w=w_{1} u w_{2}$. We will also use this notion for permutations, and say that $p$ contains $a_{1} a_{2} \ldots a_{m}$ as a factor if there is some $i$ such that $p(i+j)=a_{j}$ for all $j \in[m]$.

If $u=L^{a_{1}} R^{a_{2}} L^{a_{3}} R^{a_{4}} \ldots L^{a_{2 m-1}} R^{a_{2 m}}$ and $w$ are both words in $\{L, R\}^{*}$ with $a_{2}, a_{3}, \ldots, a_{2 m-1}>0$, we write $u \preceq_{R} w$ if and only if there exist words $w_{1}, w_{2}, \ldots, w_{2 m}$ such that $w=w_{1} w_{2} \ldots w_{2 m}$ and for all $i \in[m]$, 
(i) $w_{2 i-1}$ contains $L^{a_{2 i-1}}$ as a subword, and

(ii) $w_{2 i}$ contains $R^{a_{2 i}}$ as a factor.

For example, $L L R R \swarrow_{R} L L R L R$ (despite the fact that $L L R R \preceq L L R L R$ ), but $L L R R \preceq_{R}$ $L R L R R$. Note that like $\preceq, \preceq_{R}$ is a partial ordering on $\{L, R\}^{*}$. In fact, $\preceq$ is a refinement of $\preceq_{R}$, that is, $u \preceq w$ whenever $u \preceq_{R} w$.

Theorem 3.3 Let $p, q \in S(\{132,213\})$. Then $p$ contains a $q$ pattern if and only if $w_{B}(q) \preceq_{R} w_{B}(p)$.

Proof: Let $n=|p|$ and $k=|q|$. We induct on $n$. For $n \leq 2$ or $k \leq 2$ the theorem is easily checked, so we may assume that

$$
w_{B}(q)=w \ell_{k-2} \ell_{k-1}
$$

and

$$
w_{B}(p)=w^{\prime} \ell_{n-2}^{\prime} \ell_{n-1}^{\prime},
$$

for some $w, w^{\prime} \in\{L, R\}^{*}$ and $\ell_{k-2}, \ell_{k-1}, \ell_{n-2}^{\prime}, \ell_{n-1}^{\prime} \in\{L, R\}$. Hence $q$ is formed by inserting $k$ into $w_{B}^{-1}\left(w \ell_{k-2}\right)$ and $p$ is formed by inserting $n$ into $w_{B}^{-1}\left(w^{\prime} \ell_{n-2}^{\prime}\right)$.

First assume that $p$ contains a $q$ pattern. Then $w_{B}^{-1}\left(w^{\prime} \ell_{n-2}^{\prime}\right)$ contains a $w_{B}^{-1}\left(w \ell_{k-2}\right)$ pattern, so $w \ell_{k-2} \preceq_{R} w^{\prime} \ell_{n-2}^{\prime}$. If $w_{B}^{-1}\left(w^{\prime} \ell_{n-2}^{\prime}\right)$ contains a $q$ pattern, then by induction $w_{B}(q) \preceq_{R} w^{\prime} \ell_{n-2}^{\prime} \preceq_{R} w_{B}(p)$ and we are done. So, we may assume that $n$ plays a role in all $q$ patterns in $p$. If $p(1)=n$, then $\ell_{n-1}^{\prime}=L$, and we must have $q(1)=k$ (since $n$ must play a role in all $q$ patterns and we are assuming that there is at least one $q$ pattern in $p)$. Hence $\ell_{k-1}=L$ and $w_{B}(q) \preceq_{R} w_{B}(p)$, as desired.

Otherwise $\ell_{n-1}^{\prime}=R$. It $w_{B}(p)$ does not contain the letter $L$, then $p=12 \ldots n$, and the theorem is clearly true. So we may assume that $w_{B}(p)=u^{\prime} L R^{j}$, and thus

$$
p=(n-j)(n-j+1) \ldots n p_{j+2} p_{j+3} \ldots p_{n} .
$$

Since we are assuming that $n$ must play a role in all $q$ patterns in $p$, we must have

$$
q=(k-j)(k-j+1) \ldots k q_{j+2} q_{j+3} \ldots q_{k},
$$

so $w_{B}(q)=u L R^{j}$ for some word $u$. Furthermore, the $(n-j)$-permutation $(n-j) p_{j+2} p_{j+3} \ldots p_{n}$ must contain a $(k-j) q_{j+2} q_{j+3} \ldots q_{k}$ pattern, so by induction, $u L \preceq_{R}$ $u^{\prime} L$, and thus $w_{B}(q) \preceq_{R} w_{B}(p)$, as desired.

Now assume that $w_{B}(q) \preceq_{R} w_{B}(p)$. If $w_{B}(q) \preceq_{R} w^{\prime} \ell_{n-2}^{\prime}$, then we are done by induction, so we may assume that $w_{B}(q) \swarrow_{R} w^{\prime} \ell_{n-2}^{\prime}$, and thus $\ell_{n-1}^{\prime}=\ell_{k-1}$. Also note that we must have $w \ell_{k-2} \preceq_{R} w^{\prime} \ell_{n-2}^{\prime}$, so by induction, $w_{B}^{-1}\left(w^{\prime} \ell_{n-2}^{\prime}\right)$ contains a $w_{B}^{-1}\left(w \ell_{k-2}\right)$ pattern. If $\ell_{k-1}=\ell_{n-1}^{\prime}=L$, then $q(1)=k$ and $p(1)=n$, so $p$ contains a $q$ pattern. Otherwise $\ell_{k-1}=\ell_{n-1}^{\prime}=R, p$ contains a $(n-1) n$ factor, and $q$ contains a $(k-1) k$ factor. By assumption,

$$
w_{B}(q)=w \ell_{k-2} R \preceq_{R} w^{\prime} \ell_{n-2}^{\prime} R=w_{B}(p),
$$


but

$$
w_{B}(q) \npreceq_{R} w^{\prime} \ell_{n-2}^{\prime},
$$

and thus any $q$ pattern in $p$ must use $(n-1)$ since otherwise we could form a $q$ pattern in $w_{B}^{-1}\left(w^{\prime} \ell_{n-2}^{\prime}\right)$ and get $w_{B}(q) \preceq_{R} w^{\prime} \ell_{n-2}^{\prime}$. Therefore, since all $w_{B}\left(w \ell_{k-2}\right)$ patterns in $w_{B}^{-1}\left(w^{\prime} \ell_{n-2}^{\prime}\right)$ use $(n-1)$, and there is at least one of these patterns, $p$ contains a $q$ pattern (which uses both $n-1$ and $n$ ). $\diamond$

Almost immediately we get the following result about the relation between $s_{n}(Q)$ for sets containing $\{132,231\}$ and sets containing $\{132,213\}$.

Corollary 3.4 Let $Q \subset S(\{132,213\})$. Then for all $n$,

$$
s_{n}\left(\{132,231\} \cup w_{A}^{-1}\left(w_{B}(Q)\right)\right) \leq s_{n}(\{132,213\} \cup Q),
$$

with equality if $Q \subset S(\{132,213,123\})$

Proof: Since $\preceq_{R}$ is a refinement of $\preceq$, if $w_{B}(q) \preceq_{R} w_{B}(p)$ then $w_{B}(q) \preceq w_{B}(p)$. Therefore by Theorems 3.1 and 3.3, if $p, q \in S(\{132,213\})$ and $p$ contains a $q$ pattern, then $w_{A}^{-1}\left(w_{B}(p)\right)$ contains a $w_{A}^{-1}\left(w_{B}(q)\right)$ pattern, proving the inequality.

Now suppose that $Q \subset S(\{132,213,123\})$. Because $w_{B}(123)=R R, w_{B}(q)$ does not contain an $R R$ factor for any $q \in Q$. Hence, for all words $w \in\{L, R\}^{*}, w_{B}(q) \preceq_{R} w$ if and only if $w_{B}(q) \preceq w$.

Theorem 3.3 also allows us to construct a generating tree isomorphic to $T(Q)$ for any $Q$ containing both 132 and 213 just as we did in the case where $Q$ contains both 132 and 231 (although in this case the generating tree is slightly more complicated). We omit the explicit construction but remark that if $Q$ is an antichain (and we may always assume this) then the generating tree constructed has only finitely many labels, so again the generating function for $s_{n}(Q)$ is rational.

Next we consider sets of patterns containing 312 and 321. This is the most complicated case, but after some work we will see (Corollary 3.7) that these sets behave like sets containing 132 and 213; precisely, we will see that if $Q$ contains 312 and 321, then there is a set of patterns $Q^{\prime}$ containing 132 and 213 so that $T(Q) \cong T\left(Q^{\prime}\right)$.

As usual, we start by defining a correspondence between permutations in $S(\{312,321\})$ and words on the symbols $L$ and $R, w_{C}(p)$, and a partial ordering of these words, ${ }_{L}$. Let $w_{C}(1)=\epsilon$, and for $n>1$, assume that $p \in S_{n}(\{312,321\})$ is formed by inserting $n$ into $p^{\prime}$. Proposition 2.1 shows us that there are only two possibilities for this insertion: the next-to-last or the last position. In the former case let $w_{C}(p)=w_{C}\left(p^{\prime}\right) L$, and in the latter, $w_{C}(p)=w_{C}\left(p^{\prime}\right) R$.

We define the complement of the word $w=\ell_{1} \ell_{2} \ldots \ell_{n} \in\{L, R\}^{n}, c(w)$, to be the word whose $i$ th letter is $L$ if $\ell_{i}=R$ and is $R$ if $\ell_{i}=L$. For two words $u, w \in\{L, R\}^{*}$, we write $u \preceq_{L} w$ if and only if $c(u) \preceq_{R} c(w)$. If $u=L^{a_{1}} R^{a_{2}} L^{a_{3}} R^{a_{4}} \ldots L^{a_{2 m-1}} R^{a_{2 m}}$ with $a_{2}, a_{3}, \ldots, a_{2 m-1}>0$, this means that $u \preceq_{R} w$ if and only if there exist words $w_{1}, w_{2}, \ldots, w_{2 m}$ such that $w=w_{1} w_{2} \ldots w_{2 m}$ and for all $i \in[m]$, 
(i) $w_{2 i-1}$ contains $L^{a_{2 i-1}}$ as a factor, and

(ii) $w_{2 i}$ contains $R^{a_{2 i}}$ as a subword.

Unfortunately, $w_{C}$ and $\preceq_{L}$ do not fully capture the notion of pattern avoidance in this case. In addition, we will need the rewriting system in which any of the following operations are allowed:

(i) for $j \geq 3$, rewriting an $R^{j}$ factor with $R L^{j-1} R$,

(ii) for $j \geq 2$, rewriting an $R^{j}$ factor that occurs at the beginning of a word with $L^{j} R$,

(iii) for $j \geq 2$, rewriting an $R^{j}$ factor that occurs at the end of a word with $R L^{j}$, or

(iv) for $j \geq 1$, rewriting the word $R^{j}$ with $L^{j+1}$.

We write $w \Longrightarrow u$ if $u$ can be derived from $w$ by performing one of the operations (i)-(iv), and $w \stackrel{*}{\Longrightarrow} u$ if $u$ can be derived from $w$ by any number of operations, that is, if there are words $w_{1}, w_{2}, \ldots, w_{m-1}$ such that

$$
w=w_{0} \Longrightarrow w_{1} \Longrightarrow w_{2} \Longrightarrow \ldots \Longrightarrow w_{m}=u \text {. }
$$

For any word $w \in\{L, R\}^{*}$, define

$$
\Delta_{C}(w)=\{u: w \stackrel{*}{\Longrightarrow} u\}
$$

Note that since each of the operations (i)-(iv) decreases the number of occurrences of the letter $R$, this system is Noetherian, i.e., there is no infinite sequence of words $w_{0}, w_{1}, w_{2} \ldots$ such that

$$
w_{0} \stackrel{*}{\Longrightarrow} w_{1} \stackrel{*}{\Longrightarrow} w_{2} \stackrel{*}{\Longrightarrow} \ldots,
$$

so $\Delta_{C}(w)$ is finite for all $w$. The next lemma describes another important property of this rewriting system.

Lemma 3.5 Let $w \in\{L, R\}^{*}$ and $u \in \Delta_{C}(w)$. Then for all $j \geq 0, w R L^{j} \stackrel{*}{\Longrightarrow} u R L^{j}$, so $u R L^{j} \in \Delta_{C}\left(w R L^{j}\right)$.

Proof: Choose $m$ minimal so that there are words $w_{1}, w_{2}, \ldots, w_{m-1}$ so that

$$
w=w_{0} \Longrightarrow w_{1} \Longrightarrow w_{2} \Longrightarrow \ldots \Longrightarrow w_{m}=u \text {. }
$$

We induct on $m$. If $m=0$ then $u=w$ and the lemma is true trivially. If $m=1$, then $w \Longrightarrow u$ and we handle each operation separately. If $u$ is obtained from $w$ by either (i) or (ii) then the lemma is clearly true. If $u$ is obtained from $w$ by (iii), suppose that $w=w^{\prime} R^{i}$ where $i \geq 2$. Then we have

$$
w R L^{j}=w^{\prime} R^{i+1} L^{j} \Longrightarrow w^{\prime} R L^{i} R L^{j}=u R L^{j}
$$


by using (i). If $u$ is obtained from $w$ by (iv), then $w=R^{i}$ for some $i \geq 1$, so

$$
w R L^{j}=R^{i+1} L^{j} \Longrightarrow L^{i+1} R L^{j}=u R L^{j}
$$

by using (ii), finishing the $m=1$ case.

If $m>1$, then by induction $w R L^{j} \stackrel{*}{\Longrightarrow} w_{m-1} R L^{j}$ and $w_{m-1} R L^{j} \stackrel{*}{\Longrightarrow} u R L^{j}$ so $w R L^{j} \stackrel{*}{\Longrightarrow} u R L^{j}$, completing the proof of the lemma.

We are now ready to establish the pruning rule in this case.

Theorem 3.6 Let $p, q \in S(\{312,321\})$. Then $p$ contains a $q$ pattern if and only if $u \preceq_{L} w_{C}(p)$ for some $u \in \Delta_{C}\left(w_{C}(q)\right)$.

Proof: Let $n=|p|$ and $k=|q|$. We induct on $n$. If $n \leq 2$ or $k \leq 2$, the theorem is easily checked, so we may assume that

$$
w_{C}(q)=w \ell_{k-2} \ell_{k-1},
$$

and

$$
w_{C}(p)=w^{\prime} \ell_{n-2}^{\prime} \ell_{n-1}^{\prime},
$$

where $w, w^{\prime} \in\{L, R\}^{*}$ and $\ell_{k-2}, \ell_{k-1}, \ell_{n-2}^{\prime}, \ell_{n-1}^{\prime} \in\{L, R\}$.

First assume that $p$ contains a $q$ pattern. If $w_{C}^{-1}\left(w^{\prime} \ell_{n-2}^{\prime}\right)$ contains a $q$ pattern then we are done by induction, so we will assume that $w_{C}^{-1}\left(w^{\prime} \ell_{n-2}^{\prime}\right)$ is $q$-avoiding, and thus $n$ must play a role in every $q$ pattern in $p$. Note that $w_{C}^{-1}\left(w^{\prime} \ell_{n-2}^{\prime}\right)$ must contain a $w_{C}^{-1}\left(w \ell_{k-2}\right)$ pattern, so by induction, there is at least one word $u \in \Delta_{C}\left(w \ell_{k-2}\right)$ with $u \preceq_{L} w^{\prime} \ell_{n-2}^{\prime}$.

If $p(n)=n$ (so $\ell_{n-1}^{\prime}=R$ ), then since there is at least one $q$ pattern in $p$ and all such patterns must involve the element $n$ we have $q(k)=k$ (so $\ell_{k-1}=R$ ). Hence $u R \preceq_{L} w_{C}(p)$ and since $u \in \Delta_{C}\left(w \ell_{k-2}\right)$, by Lemma 3.5, $u R \in \Delta_{C}\left(w \ell_{k-2} R\right)=\Delta_{C}\left(w_{C}(q)\right)$.

Otherwise $p(n-1)=n$ and thus $\ell_{n-1}^{\prime}=L$. There are two possibilities: either $p(n-2)=n-1\left(\right.$ so $\left.\ell_{n-2}^{\prime}=L\right)$, or $p(n)=n-1$ (so $\left.\ell_{n-2}^{\prime}=R\right)$. In either case, because $n-1$ and $n$ are adjacent in $p$ and $w_{C}^{-1}\left(w^{\prime} \ell_{n-2}^{\prime}\right)$ is $q$-avoiding, every $q$ pattern in $p$ must use $(n-1)$ as well as $n$. In the latter case, this implies that $q(k)=k-1$ and $q(k-1)=k$, and thus $w_{C}(q)=w R L$, and again using Lemma 3.5 we are done.

The former case, where $p(n-1)=n, p(n-2)=n-1$, and thus $w_{C}(p)=w^{\prime} L L$ is slightly more difficult. First, if $w_{C}(p)=L^{n-1}$ then $p=23 \ldots n 1$, so $p$ only contains patterns of the form $12 \ldots(j+1)$ (with $j<n-1)$ and $23 \ldots(j+1) 1$ (with $j \leq n-1)$. If $q=12 \ldots(j+1)$ for some $1 \leq j<n-1$, then $w_{C}(q)=R^{j}$ and by applying operation (iv) we see that $L^{j+1} \in \Delta_{C}\left(w_{C}(q)\right)$. We are now done because $L^{j+1} \preceq_{L} w_{C}(p)$. In the other case, $q=23 \ldots(j+1) 1$ for some $1 \leq j \leq n-1$, so $w_{C}(q)=L^{j} \preceq_{L} w_{C}(p)$. Therefore we may now assume that $w^{\prime}$ contains the letter $R$, so let $w_{C}(p)=v^{\prime} R L^{j}$, where $v \in\{L, R\}^{n-j-2}$. Then

$$
p=p_{1} p_{2} \ldots p_{n-j-1}(n-j+1)(n-j+2) \ldots n(n-j) .
$$


If there is a $q$ pattern in $p$ that uses the element $(n-j)$, then we must have

$$
q=q_{1} q_{2} \ldots q_{k-j-1}(k-j+1)(k-j+2) \ldots k(k-j),
$$

so $w_{C}^{-1}\left(v^{\prime}\right)=p_{1} p_{2} \ldots p_{n-j-1}$ contains a $q_{1} q_{2} \ldots q_{k-j-1}$ pattern. Hence by induction there is some $v \in \Delta_{C}\left(w_{C}\left(q_{1} q_{2} \ldots q_{k-j-1}\right)\right)$ with $v \preceq_{L} v^{\prime}$ and by Lemma 3.5,

$$
v R L^{j} \in \Delta_{C}\left(w_{C}\left(q_{1} q_{2} \ldots q_{k-j-1}\right) R L^{j}\right)=\Delta_{C}\left(w_{C}(q)\right)
$$

and thus we are done because $v R L^{j} \preceq_{L} v^{\prime} R L^{j}$.

Otherwise none of the $q$ patterns in $p$ use the element $(n-j)$, so

$$
w_{C}^{-1}\left(v^{\prime} R^{j}\right)=\operatorname{red}\left(p_{1} p_{2} \ldots p_{n-j-1}(n-j+1)(n-j+2) \ldots n\right)
$$

contains a $q$ pattern. By induction this means that there is some $u \in \Delta_{C}\left(w_{C}(q)\right)$ with $u \preceq_{L} v^{\prime} R^{j}$. Hence $u=u^{\prime} R^{j}$, and thus

$$
w_{C}(q) \stackrel{*}{\Longrightarrow} u=u^{\prime} R^{j} \Longrightarrow u^{\prime} R L^{j} \preceq_{L} v^{\prime} R L^{j}=w_{C}(p),
$$

by applying operation (iii). Therefore we are finished with this direction of the proof.

Now assume that there is some $u \in \Delta_{C}\left(w_{C}(q)\right)$ with $u \preceq_{L} w_{C}(p)$. We will show that $p$ contains a $q$ pattern by first showing that $p$ contains a $w_{C}^{-1}(u)$ pattern and then showing that $w_{C}^{-1}(u)$ contains a $q$ pattern.

Note that since each of the operations (i)-(iv) is length increasing, $m \geq k \geq 2$. Let

$$
u=w^{\prime \prime} \ell_{m-2}^{\prime \prime} \ell_{m-1}^{\prime \prime}
$$

where $w^{\prime \prime} \in\{L, R\}^{*}$ and $\ell_{m-2}^{\prime \prime}, \ell_{m-1}^{\prime \prime} \in\{L, R\}$. As usual we may assume by induction that $u \swarrow_{L} w^{\prime} \ell_{n-2}^{\prime}$, so $\ell_{n-1}^{\prime}=\ell_{m-1}^{\prime}$. Since we must have $w^{\prime \prime} \ell_{m-2}^{\prime \prime} \preceq_{L} w^{\prime} \ell_{n-2}^{\prime}$, by induction we see that $w_{C}^{-1}\left(w^{\prime} \ell_{n-2}^{\prime}\right)$ contains a $w_{C}^{-1}\left(w^{\prime \prime} \ell_{m-2}^{\prime \prime}\right)$ pattern. Hence if $\ell_{n-1}^{\prime}=\ell_{m-1}^{\prime \prime}=R$, so $p(n)=n$ and $w_{C}^{-1}(u)(m)=m$, then $p$ contains a $w_{C}^{-1}(u)$ pattern as desired.

This leaves us with the case where $\ell_{n-1}^{\prime}=\ell_{m-1}^{\prime \prime}=L$. If $\ell_{m-2}^{\prime \prime}=R$ then $w_{C}^{-1}(u)$ ends with an $n(n-1)$ factor. There must be at least one occurrence of the letter $R$ in $w_{C}(p)$, and thus we may write

$$
w_{C}(p)=v^{\prime} R L^{j}
$$

Then $w^{\prime \prime} R \preceq_{L} v^{\prime} R$ so by induction $w_{C}^{-1}\left(v^{\prime} R\right)$ contains a $w_{C}^{-1}\left(w^{\prime \prime} R\right)$ pattern, and from here it is clear that $p$ contains a $w_{C}^{-1}(u)$ pattern.

If $\ell_{m-2}^{\prime \prime}=L$, then

$$
u=w^{\prime \prime} L L \preceq_{L} w^{\prime} \ell_{n-2}^{\prime} L=w_{C}(p),
$$

so since we have assumed that $u \swarrow_{L} w^{\prime} \ell_{n-2}^{\prime}$, we must have $\ell_{n-2}^{\prime}=L$. Therefore $p$ ends with an $(n-1) n x^{\prime}$ pattern for some $x^{\prime} \in[n-2]$ and $w_{C}^{-1}(u)$ ends with an $(m-1) m x^{\prime \prime}$ pattern for some $x^{\prime \prime} \in[m-2]$. If there is a $w_{C}^{-1}\left(w^{\prime \prime} L\right)$ pattern in $w_{C}^{-1}\left(w^{\prime}\right)$, then we are done by induction, so we may assume that all the $w_{C}^{-1}\left(w^{\prime \prime} L\right)$ patterns in $w_{C}^{-1}\left(w^{\prime} L\right)$ (we have remarked earlier that there must be at least one of these) use the element $n-1$. This shows that $p$ contains a $w_{C}^{-1}(u)$ pattern, completing this part of the proof. 
It remains only to show that $w_{C}^{-1}(u)$ contains a $q$ pattern for all $u \in P\left(w_{C}(q)\right)$. Choose $m$ minimal so that there are words $w_{1}, w_{2}, \ldots, w_{m-1}$ so that

$$
w_{C}(q)=w_{0} \Longrightarrow w_{1} \Longrightarrow w_{2} \Longrightarrow \ldots \Longrightarrow w_{m}=u \text {. }
$$

We induct on $m$. If $m=0$ then $w_{C}^{-1}(u)=q$ and the claim is true. If $m=1$, then $w_{C}(q) \Longrightarrow u$ and we examine each operation separately.

Suppose that $u$ is obtained from $w_{C}(q)$ by (iv). Then

$$
\begin{aligned}
q & =12 \ldots k, \\
w_{C}^{-1}(u) & =23 \ldots(k+1) 1,
\end{aligned}
$$

and we get $q$ from $w_{C}^{-1}(u)$ by removing 1 and reducing.

If $u$ is obtained from $w_{C}(q)$ by (iii), then

$$
w_{C}(q)=w_{1} R^{j} \Longrightarrow w_{1} R L^{j}=u
$$

SO

$$
\begin{aligned}
q & =w_{C}^{-1}\left(w_{1}\right)(k-j+1)(k-j+2) \ldots k, \\
w_{C}^{-1}(u) & =w_{C}^{-1}\left(w_{1}\right)(k-j+2)(k-j+3) \ldots(k+1)(k-j+1),
\end{aligned}
$$

and to get $q$ from $w_{C}^{-1}(u)$ we just remove $k-j+1$ and reduce.

If $u$ is obtained from $w_{C}(q)$ by (ii), then

$$
w_{C}(q)=R^{j} w_{1} \Longrightarrow w_{1} L^{j} R w_{1}=u
$$

and $q$ is equal to the reduction of the permutation obtained from $w_{C}^{-1}(u)$ by removing the element 1.

Finally, if $u$ is obtained from $w_{C}(q)$ by $(\mathrm{i})$, then

$$
w_{C}(q)=w_{1} R^{j} w_{2} \Longrightarrow w_{1} R L^{j-1} R w_{2}=u \text {. }
$$

and $q$ is equal to the reduction of the permutation obtained by removing the element $\left|w_{1}\right|+1$ from $w_{C}^{-1}(u)$, so $w_{C}^{-1}(u)$ contains a $q$ pattern.

If $m>1$, then by induction $w_{C}^{-1}\left(w_{m-1}\right)$ contains a $q$ pattern and $w_{C}^{-1}(u)$ contains a $w_{C}^{-1}\left(w_{m-1}\right)$ pattern, so $w_{C}^{-1}(u)$ contains a $q$ pattern, completing the proof.

Immediately from Theorems 3.3 and 3.6, we get the following result about Wilfequivalence.

Corollary 3.7 Let $Q \subset S(\{312,321\})$. Then for all $n$,

$$
s_{n}(\{312,321\} \cup Q)=s_{n}\left(\{132,213\} \cup w_{B}^{-1}\left(c\left(\Delta_{C}\left(w_{C}(Q)\right)\right)\right)\right) .
$$


Therefore for any set of patterns $Q$ containing both 312 and $321, s_{n}(Q)$ has a rational generating function, and we can find this generating function by using Corollary 3.7 and then constructing the generating tree alluded to after Theorem 3.3.

We have only one more symmetry class to consider, sets containing 132 and 321. From Theorem 3.1 we see that

$$
T(\{132,321\}) \cong T(\{132,231,4213\})
$$

because $w_{A}(4213)=L R L$. In fact, we will see that $T(\{132,321\})$ prunes much like $T(\{132,231,4213\})$.

As usual, let $w_{D}(1)=\epsilon$, and for $n>1$ assume that $p \in S_{n}(\{132,321\})$ is formed by inserting $n$ into $p^{\prime}$. We define $w_{D}(p)$ by $w_{D}(p)=w_{D}\left(p^{\prime}\right) R$ if $p(n)=n$, and $w_{D}(p)=$ $w_{D}\left(p^{\prime}\right) L$ otherwise (in this case, either $p=n 1 \ldots(n-1)$ or $n$ was inserted right after $n-1)$. By Proposition 2.2 the image of $w_{D}$ is precisely the set $\left\{w \in\{L, R\}^{*}: L R L \npreceq w\right\}$.

We will not need a new partial order for this case, but we do need to define another rewriting system. In this system only one operation is allowed: rewriting the word $R^{i+j}$ as $L^{i} R^{j}$. Let

$$
\Delta_{D}(w)=\{u: w \stackrel{*}{\Longrightarrow} u\}
$$

SO

$$
\Delta_{D}(w)= \begin{cases}\{w\} & \text { if } L \preceq w \\ \left\{L^{i} R^{|w|-i}: 0 \leq i \leq|w|\right\} & \text { if } L \npreceq w .\end{cases}
$$

Theorem 3.8 Let $p, q \in S(\{132,321\})$. Then $p$ contains a $q$ pattern if and only if $u \preceq w_{D}(p)$ for some $u \in \Delta_{D}\left(w_{D}(q)\right)$.

Proof: As we remarked above, because $p, q \in S(\{132,321\}), L R L \npreceq w_{D}(p), w_{D}(q)$. This means that $w_{D}(p)=R^{a_{1}} L^{a_{2}} R^{a_{3}}$ and $w_{D}(q)=R^{b_{1}} L^{b_{2}} R^{b_{3}}$ for some integers $a_{1}, a_{2}, a_{3}$, $b_{1}, b_{2}, b_{3} \geq 0$. Furthermore, we have that

$$
\begin{aligned}
& w_{D}^{-1}\left(R^{i} L^{j} R^{k}\right)= \\
& \quad(i+2)(i+3) \ldots(i+j+1) 12 \ldots(i+1)(i+j+2)(i+j+3) \ldots(i+j+k+1) .
\end{aligned}
$$

So $w_{D}^{-1}\left(R^{i} L^{j} R^{k}\right)$ consists of three (possibly empty) increasing factors of respective lengths $b, a+1$, and $c$ such that every element in the first increasing factor is greater than all elements in the second, but less than all elements in the third.

First, if $q$ is not $12 \ldots k$, then $L \preceq w_{D}(q)$ and clearly $p$ has a $q$ pattern if and only if $b_{i} \leq a_{i}$ for all $i \in[3]$. Since $\Delta_{D}\left(w_{D}(q)\right)=\left\{w_{D}(q)\right\}$ in this case, we are done.

Otherwise $q=12 \ldots k$ (so $w_{D}(q)=R^{k-1}$ ) and we need to consider several different kinds of $q$ patterns in $p$. First, $p$ could have a $q$ pattern in the $12 \ldots\left(a_{1}+1\right)$ factor. This occurs if and only if $k-1 \leq a_{1}$, i.e., if and only if $R^{k-1} \preceq R^{a_{1}}$. Secondly, we could have a $q$ pattern in the $\left(a_{1}+a_{2}+2\right)\left(a_{1}+a_{2}+3\right) \ldots\left(a_{1}+a_{2}+a_{3}+1\right)$ factor. This occurs if and only if $k-1 \leq a_{3}$, which is if and only if $R^{k-1} \preceq R^{a_{3}}$. We could also have a $q$ pattern formed using elements from both of these factors, which occurs if and only if $R^{k-1} \preceq R^{a_{1}} R^{a_{3}}$. All other $q$ patterns must use the $\left(a_{1}+2\right)\left(a_{1}+3\right) \ldots\left(a_{1}+a_{2}+1\right)$ factor. 
Hence these patterns cannot use the $12 \ldots\left(a_{1}+1\right)$ factor, so we have such patterns if and only if $L^{i} R^{k-1-i} \preceq L^{a_{2}} R^{a_{3}}$ for some $i \geq 0$. Putting this together with our expression for $\Delta_{D}\left(w_{D}(q)\right)$, the theorem is proved.

Theorem 3.8 gives us our last result about Wilf-equivalence.

Corollary 3.9 Let $Q \subset S(\{132,321\})$. Then for all $n$,

$$
s_{n}(\{132,321\} \cup Q)=s_{n}\left(\{132,231,4213\} \cup w_{A}^{-1}\left(\Delta_{D}\left(w_{D}(Q)\right)\right)\right) .
$$

We conclude this section by collecting our various results about rational generating functions.

Theorem 3.10 Let $Q$ be any set of patterns that contains two elements of $S_{3}$. Then $T(Q)$ is isomorphic to a generating tree with only finitely many labels, and thus the generating function for $s_{n}(Q)$ is rational.

\section{Interesting sets of restrictions}

Simion and Schmidt [18] showed that $s_{n}(\{123,132,213\})=F_{n+1}$, the $n+1$ st Fibonacci number. Egge [6] generalized this to show that for all $n \geq 0$ and $k \geq 2$,

$$
s_{n}(\{123,132,(k-1)(k-2) \ldots 1 k\})=s_{n}(\{132,213,12 \ldots k\})=F_{n+1}^{(k-1)},
$$

where $F_{n}^{(k)}$ denotes the $n$th $k$-generalized Fibonacci number defined by $F_{n}^{(k)}=0$ for $n \leq 0, F_{1}^{(k)}=1$, and $F_{n}^{(k)}=\sum_{i=1}^{k} F_{n-i}^{(k)}$ for $n \geq 2$. This follows easily from our work in the previous section. First, note that $\{123,132,(k-1)(k-2) \ldots 1 k\}$ and $\{312,321,23 \ldots k 1\}$ are in the same symmetry class. Also, $w_{B}(12 \ldots k)=R^{k-1}, w_{C}(23 \ldots k 1)=L^{k-1}$, and $\Delta_{C}\left(L^{k-1}\right)=\left\{L^{k-1}\right\}$, so $\{123,132,(k-1)(k-2) \ldots 1 k\}$ and $\{132,213,12 \ldots k\}$ are Wilfequivalent by Corollary 3.7 .

Hence we need only compute $s_{n}(\{132,213,12 \ldots k\})$. Because $w_{B}(12 \ldots k)=R^{k-1}$, Theorem 3.3 tells us that there is a bijection between $S_{n}(\{132,213,12 \ldots k\})$ and the set of words in $\{L, R\}^{n-1}$ that do not contain an $R^{k-1}$ factor. It is well-known that the number of such words is $F_{n+1}^{(k-1)}$.

Egge [6] performs another calculation that follows easily from our previous work. It is

$$
s_{n}(\{132,213,23 \ldots k 1\})=\sum_{i=1}^{n} F_{i}^{(k-2)} .
$$

Since $w_{B}(23 \ldots k 1)=L R^{k-2}$, we get

$$
\begin{aligned}
s_{n}(\{132,213,23 \ldots k 1\}) & =s_{n-1}\left(\left\{132,213, w_{B}^{-1}\left(L R^{k-2}\right)\right\}\right)+s_{n-1}\left(\left\{132,213, w_{B}^{-1}\left(R^{k-2}\right)\right\}\right), \\
& =s_{n-1}(\{132,213,23 \ldots k 1\})+s_{n-1}(\{132,213,12 \ldots(k-1)\}), \\
& =s_{n-1}(\{132,213,23 \ldots k 1\})+F_{n}^{(k-2)},
\end{aligned}
$$


by our previous calculation, and now (1) follows. Notice that the differences of $s_{n}(\{132,213,23 \ldots k 1\})$ satisfy the $(k-2)$-generalized Fibonacci recurrence. Inspired by this and a calculation that is not aided by our work (that the $(k-3)$ rd differences of $s_{n}(\{132,2341, k(k-1) \ldots 4213\})$ satisfy the Fibonacci recurrence), Egge asked: for all $i \geq 0$ and $k \geq 1$, is there a set of patterns $Q_{i, k}$ such that the $i$ th differences of $s_{n}\left(Q_{i, k}\right)$ satisfy the $k$-generalized Fibonacci recurrence? We answer this in the affirmative, with

$$
Q_{i, k}=\{132,213,(i+1)(i+2) \ldots(i+k+1) i(i-1) \ldots 1\} .
$$

The desired result follows easily from induction on $i$ and the fact that

$$
w_{B}((i+1)(i+2) \ldots(i+k+1) i(i-1) \ldots 1)=L^{i} R^{k}
$$

SO

$$
s_{n}\left(Q_{i, k}\right)=s_{n-1}\left(Q_{i, k}\right)+s_{n-1}\left(Q_{i-1, k}\right),
$$

for $i, n \geq 1$ (we have already settled the $i=0$ case above).

We can also get a sum of a bounded number of generalized Fibonacci numbers by adding another pattern to sets we have already considered:

$$
s_{n}(\{132,213,23 \ldots k 1,12 \ldots(j+1)\})=\sum_{i=1}^{j} F_{i}^{(k-2)} .
$$

Furthermore, we can find sets that (for $n$ large enough) give us any constant function we would like:

$$
s_{n}(\{132,213,231,12 \ldots(k+1)\})=\min \{n, k\}
$$

for all $n$.

\section{When $s_{n}(Q)$ is (essentially) a polynomial}

Theorem 3.10 tells us that there is a finitely-labeled generating tree isomorphic to $T(Q)$ whenever $\left|Q \cap S_{3}\right| \geq 2$. In addition to giving rise to rational generating functions, finitelylabeled generating trees are nice because they are equivalent to deterministic informationless Lindenmayer systems, or D0L-systems for short (see Ferrari et al. [7] for details). The reader is referred to Rozenberg and Salomaa [16] for more information on D0L-systems. We will make use only of the following result of Salomaa, which we have translated into generating tree terminology.

Theorem 5.1 [17] Let $T$ be a finitely-labeled generating tree. If there is a node, say $x \in T$, such that $T_{\leq x}$ contains two nodes on the same level with the same label as $x$, then the level sums of $T$ are (clearly) exponential. Otherwise the level sums of $T$ are bounded by a polynomial. 
It is relatively easy to see that if $\emptyset \neq Q \subset S(\{132,231\})$, then the generating tree isomorphic to $T(\{132,231\} \cup Q)$ that we constructed in Section 3 satisfies the hypotheses of Theorem 5.1: the $i$ th component of $d_{\ell}(\vec{a})$ is at least the $i$ th component of $\vec{a}$, and since $Q \neq \emptyset$, for some $i \in|Q|$ the $i$ th component of either $d_{L}(\vec{a})$ or $d_{R}(\vec{a})$ is strictly greater than the $i$ th component of $\vec{a}$.

If $T$ is a generating tree and $x$ and $y$ are nodes in $T$, then $x$ and $y$ can not share a label if $T_{\leq x} \neq T_{\leq y}$, but they can share a label if $T_{\leq x} \cong T_{\leq y}$. Let $\lambda(T)$ denote the cardinality of the largest set of nodes $X \subset T$ such that $T_{\leq x} \neq T_{\leq y}$ whenever $x, y \in X$ with $x \neq y$. In other words, $\lambda(T)$ is the minimum number of labels needed for $T$. Note that if $y$ is a descendant of $x$ in $T$, then $\lambda\left(T_{\leq y}\right) \leq \lambda\left(T_{\leq x}\right)$.

Theorem 5.2 Suppose that the generating tree $T$ has polynomially bounded level sums and the additional property that if $y$ is a child of $x$ then $\lambda\left(T_{\leq y}\right)=\lambda\left(T_{\leq x}\right)$ only if $T_{\leq y} \cong T_{\leq x}$. Then there is a polynomial $p(n)$ of degree at most $\lambda(T)$ such that $T$ has precisely $p(n)$ nodes on level $n$ for all $n \geq \lambda(T)$.

Proof: Let $p_{T}(n)$ denote the number of nodes on level $n$ of $T$. We induct on $\lambda(T)$. If $\lambda(T)=1$, then since the level sums of $T$ are bounded by a polynomial, we must have $p_{T}(n)=1$ for all $n \geq 1$. Now assume that $\lambda(T) \geq 2$ and that the children of the root node are $x_{1}, x_{2}, \ldots, x_{k}$. Hence for all $n \geq 2$,

$$
p_{T}(n)=\sum_{i=1}^{k} p_{T_{\leq x_{i}}}(n-1) .
$$

If $\lambda\left(T_{\leq x_{1}}\right)<\lambda(T)$ for all $i \in[k]$, then the theorem follows by induction. Otherwise, without loss assume that $T_{\leq x_{1}} \cong T$. Note that since $T$ has polynomially bounded level sums, this is the only such child. Therefore we get that

$$
p_{T}(n)-p_{T}(n-1)=\sum_{i=2}^{k} p_{T_{\leq x_{i}}}(n-1),
$$

and we are again done by induction. $\diamond$

Applying this to our situation gives the following result.

Corollary 5.3 Let $\emptyset \neq\left\{q_{1}, q_{2}, \ldots, q_{m}\right\} \subset S(\{132,231\})$. Then there is a polynomial of degree at most $\left(\left|q_{1}\right|-1\right)\left(\left|q_{2}\right|-1\right) \ldots\left(\left|q_{m}\right|-1\right)$ that agrees with $s_{n}\left(\left\{132,231, q_{1}, q_{2}, \ldots, q_{m}\right\}\right)$ for all $n \geq\left(\left|q_{1}\right|-1\right)\left(\left|q_{2}\right|-1\right) \ldots\left(\left|q_{m}\right|-1\right)$.

Note that by our previous work on Wilf-equivalence, similar results hold in two other cases:

(1) By Corollary 3.4, if $\emptyset \neq\left\{q_{1}, q_{2}, \ldots, q_{m}\right\} \subset S(\{132,213,123\})$ then there is a polynomial of degree at most $\left(\left|q_{1}\right|-1\right)\left(\left|q_{2}\right|-1\right) \ldots\left(\left|q_{m}\right|-1\right)$ that agrees with $s_{n}\left(\left\{132,213, q_{1}, q_{2}, \ldots, q_{m}\right\}\right)$ for all $n \geq\left(\left|q_{1}\right|-1\right)\left(\left|q_{2}\right|-1\right) \ldots\left(\left|q_{m}\right|-1\right)$.

(2) By Corollary 3.9, if $132,321 \in Q$ then there is a polynomial that agrees with $s_{n}(Q)$ for all large $n$. 


\section{Conclusion}

In [15], Noonan and Zeilberger studied permutations that contain patterns a prescribed (possibly non-zero) number of times. In particular they conjectured that the number of $n$ permutations with exactly $r_{1}, r_{2}, \ldots, r_{j}$ copies of the patterns $q_{1}, q_{2}, \ldots, q_{j}$ is P-recursive in $n$. Atkinson [1] showed using Inclusion-Exclusion and some simple counting arguments that there are finite sets of patterns $Q_{1}, Q_{2}, \ldots, Q_{k}$ so that the number of the aformentioned permutations is an integral linear combination of $s_{n}\left(Q_{1}\right), s_{n}\left(Q_{2}\right), \ldots, s_{n}\left(Q_{k}\right)$. Hence the Noonan-Zeilberger Conjecture is equivalent to the seemingly weaker conjecture of Gessel [8] that $s_{n}(Q)$ is P-recursive for all finite sets of patterns $Q$. If $r_{1}=r_{2}=0$, then Atkinson's argument shows that the number of these permutations is equal to an integral linear combination of $s_{n}\left(\left\{q_{1}, q_{2}\right\} \cup Q_{1}\right), s_{n}\left(\left\{q_{1}, q_{2}\right\} \cup Q_{2}\right), \ldots, s_{n}\left(\left\{q_{1}, q_{2}\right\} \cup Q_{k}\right)$ for some sets of patterns $Q_{1}, Q_{2}, \ldots Q_{k}$. Hence by Theorem 3.10, the Noonan-Zeilberger Conjecture is true whenever $r_{1}=r_{2}=0$ and $q_{1}, q_{2} \in S_{3}$ with $q_{1} \neq q_{2}$. Moreover, Atkinson gives an upper bound on the lengths of the permutations in $Q_{1}, Q_{2}, \ldots, Q_{k}$, so together with our results from Section 3, we get an algorithm to compute the number of these permutations in the case where $r_{1}=r_{2}=0, q_{1}, q_{2} \in S_{3}$, and $q_{1} \neq q_{2}$. This is similar to the work of Mansour [14], who computed generating functions for the number of $n$-permutations that avoid two distinct patterns of length three and contain exactly one copy of another pattern of any length.

It is natural to wonder what other pattern avoidance trees $T(Q)$ have nice pruning rules. If the pruning rules are to look like the rules presented here then the tree must have bounded degrees, i.e., there must be a constant $d$ such that every permutation $p \in S(Q)$ has at most $d$ children in $T(Q)$. This forces us to have, for some $j, k \geq 0$, both a child of $12 \ldots j$ and a child of $k \ldots 21$ in $Q$ because otherwise for all $n$ either $12 \ldots n$ or $n \ldots 21$ will have $n+1$ children and thus the tree will not have bounded degrees. In fact, Kremer and Shiu [11] showed that this condition is sufficient.

Theorem 6.1 [11] The pattern-avoidance tree $T(Q)$ has bounded degrees if and only if for some $j, k \geq 0, Q$ contains a child of $12 \ldots j$ and a child of $k \ldots 21$.

For a start, it would be nice to know if all the pattern-avoidance trees with bounded degrees are isomorphic to finitely labeled generating trees. We have seen in Section 3 that this is true if $\left|Q \cap S_{3}\right| \geq 2$, and Kremer and Shiu [11] found it to be true when $Q$ contains precisely two distinct elements of $S_{4}$.

Acknowledgment. I am grateful to Doron Zeilberger for his helpful comments.

\section{References}

[1] M. D. Atkinson, Restricted permutations, Discrete Math. 195 (1999), 27-38.

[2] M. D. Atkinson, M. M. Murphy, and N. Ruškuc, Partially well-ordered closed sets of permutations, Order 19 (2002), 101-113. 
[3] C. Banderier, M. Bousquet-Mélou, A. Denise, P. Flajolet, D. Gardy, and D. GouyouBeauchamps, Generating functions for generating trees, Discrete Math. 246 (2002), $29-55$.

[4] S. Billey, W. Jockusch, and R. P. Stanley, Some combinatorial properties of Schubert polynomials, J. Algebraic Combin. 2 (1993), 345-374.

[5] F. R. K. Chung, R. L. Graham, V. E. Hoggatt Jr., and M. Kleiman, The number of Baxter permutations, J. Combin. Theory Ser. A 24 (1978), 382-394.

[6] E. S. Egge, Restricted permutations related to Fibonacci numbers and $k$-generalized Fibonacci numbers, arXiv:math.CO/0109219.

[7] L. Ferrai, E. Pergola, R. Pinzani, and S. Rinaldi, An algebraic characterization of the set of succession rules, Theoret. Comput. Sci. 281 (2002), 351-367.

[8] I. Gessel, Symmetric functions and P-recursiveness, J. Combin. Theory Ser. A 53 (1990), 257-285.

[9] O. Guibert, Permutations sans sous séquence interdite, Mémoire de Diplôme d'Etudes Aprrofondies de L'Université Brodeaux I, 1992.

[10] D. E. Knuth, "The Art of Computer Programming, Volume 1," Addison-Wesley, Reading MA, 1973.

[11] D. Kremer and W. Shiu, Finite transition matrices for permutations avoiding pairs of length four patterns, preprint.

[12] P. A. MacMahon, "Combinatory Analysis," Chelsea NY, 1960 (Originally published by Cambridge University Press, London, 1915/16).

[13] T. Mansour, Permutations avoiding a pattern from $S_{k}$ and at least two patterns from $S_{3}$, Ars Combin. 62 (2002), 227-239.

[14] T. Mansour, Permutations containing a pattern exactly once and avoiding at least two patterns of three letters, arXiv:math.CO/0202007.

[15] J. Noonan and D. Zeilberger, The enumeration of permutations with a prescribed number of "forbidden" patterns, Adv. in Appl. Math 17 (1996), 381-407.

[16] G. Rozenberg and A. Salomaa, "The Mathematical Theory of L Systems," Academic Press, London, 1980.

[17] A. Salomaa, On exponential growth in Lindenmayer systems, Indag. Math. 35 (1973), $23-30$.

[18] R. Simion and F. W. Schmidt, Restricted permutations, European J. Combin. 6 (1985), 383-406. 
[19] J. West, Generating trees and forbidden subsequences, Discrete Math. 157 (1996), 363-374.

[20] J. West, Generating trees and the Catalan and Schröder numbers, Discrete Math. 146 (1995), 247-262. 